\title{
EMERGENCE OF CONSENSUS OF MULTI-AGENTS SYSTEMS ON TIME SCALES
}

\author{
EWA SCHMEIDEL, URSZULA OSTASZEWSKA, AND MAŁGORZATA ZDANOWICZ
}

Received 12 October, 2018

\begin{abstract}
In this paper an emergence of leader-following consensus on arbitrary time scales is investigated. It means that the step size is not necessarily constant but it is a function of time. We propose and prove conditions ensuring a leader-following consensus for discrete time scales. The presented results are illustrated by numerical examples.
\end{abstract}

2010 Mathematics Subject Classification: 34N05; 34D20; 93C10

Keywords: time scales, leader-following problem, emergence of consensus, multi-agent systems, networked control systems, graph, Laplacian

\section{INTRODUCTION}

Flocking is a form of collective behaviour of a large number of interacting agents with a common group objective. Such a group is known as a multi-agent system. Examples of these multi-agent systems include crowds, bees, ants, birds, fish and penguins. Often in the group of agents there is one special agent which is called the leader. The leader is an agent, whose motion is independent of all the other agents and is followed by all the other ones. In this paper, we study model of behaviour of multi-agent system with the leader. The emergence of consensus is an important topic in multi-agent systems. The main idea is to drive a team of agents to reach an agreement on a certain issue by negotiating with the leader and with their neighbours. Although, each individual agent has limited processing power, the interconnected system as a whole can perform complex tasks in a coordinated fashion. Agents share their states with their neighbours via a chaotic communication network. The model describes the information transfers between agents particularly between agents and the leader. The model could be applicable for describing negotiation process in a group of number of people in which one of them is more important than the others, as well as for describing movement of group of animals with the leader. The origins of the investigation of the leader-following problem date back to the 1970s.

The first author was supported by the Polish National Science Center grant on the basis of decision DEC-2014/15/B/ST7/05270. 
In 1974 [9], DeGroot studied explicitly described process leading to the consensus. The general objective of the paper published by French in 1977 [10], was to enhance the understanding of organizational power by constructing a formal theory of power as a process characterizing the relations among organizations. In 2000, Krause [15] proposed the model of a group of experts who have to make a joint assessment of a certain magnitude. Each of the experts has his own opinion but is open to some extent to revise it when being informed about the opinions of all the other experts. Coordination of groups of mobile autonomous agents using nearest neighbour rules was studied by Jadbabaie et al. in [14]. In [3,4], Blondel et al. investigated Krause's multi-agent consensus model with state-dependent connectivity. Girejko et al. studied Krause's model of opinion dynamics on isolated time scales [11,13]. In 2007, Cucker and Smale [7,8] published two papers devoted to an emergent behaviour in flocks. The authors provided the model describing the evolution of a flock for both continuous and discrete time. Cucker-Smale model on isolated time scales is studied by Girejko et al. in [13]. In 2015, Wang et al. [19] studied the leader-following consensus of discrete time linear multi-agent systems with communication noises. Recently, in 2018, Girejko, Machado, Malinowska, and Martins, have published some results for consensus in the Cucker-Smale type model on isolated time scales [12]. In [2], using the time scale theory, Babenko et al. investigated the leader-follower consensus problem for high-order multi-agent systems with inherent non-linear dynamics evolving on an arbitrary time domain. The authors obtained some sufficient conditions to guarantee that the tracking errors exponentially converge to zero using the concept of matrix-valued Lyapunov functions.

Notice that an interaction topology in the multi-agent system is modelled by undirected or directed graph. Our analysis framework is based on tools from matrix theory, algebraic graph theory and time scales theory.

\section{BASIS OF TIME SCALES CALCULUS}

A time scale is a model of time $[1,5,6]$, where the step size is a function of time. From mathematical point of view it is an arbitrary nonempty closed subset $\mathbb{T}$ of the set $\mathbb{R}$ of real numbers.

The mapping $\sigma: \mathbb{T} \rightarrow \mathbb{T}$, defined by $\sigma(t)=\inf \{s \in \mathbb{T}: s>t\}$ with inf $\varnothing=\sup \mathbb{T}$, is called the forward jump operator. Similarly, we define the backward jump operator $\rho: \mathbb{T} \rightarrow \mathbb{T}$ by $\rho(t)=\sup \{s \in \mathbb{T}: s<t\}$ with $\sup \varnothing=\inf \mathbb{T}$. The following classification of points is used within the theory: a point $t \in \mathbb{T}$ is called right-dense, rightscattered, left-dense and left-scattered if $\sigma(t)=t$ (for $t<\sup \mathbb{T}$ ), $\sigma(t)>t, \rho(t)=t$ (for $t>\inf \mathbb{T}$ ) and $\rho(t)<t$, respectively. We say that $t$ is isolated if $\rho(t)<t<\sigma(t)$, and that $t$ is dense if $\rho(t)=t=\sigma(t)$. The function $\mu: \mathbb{T} \rightarrow[0, \infty)$ is defined by $\mu(t)=\sigma(t)-t$ and called the graininess function. The delta (or Hilger) derivative of 
$f: \mathbb{T} \rightarrow \mathbb{R}$ at a point $t \in \mathbb{T}^{\kappa}$, where

$$
\mathbb{T}^{\kappa}:=\left\{\begin{array}{l}
\mathbb{T} \backslash(\rho(\sup \mathbb{T}), \sup \mathbb{\mathbb { T }}] \text { if } \sup \mathbb{T}<\infty \\
\mathbb{T} \text { if } \sup \mathbb{\mathbb { T }}=\infty
\end{array},\right.
$$

is defined in the following way.

Definition 1 ([5]). The delta derivative $f^{\Delta}(t)$ is the number (provided it exists) with the property that given any $\varepsilon>0$, there is a neighbourhood $U$ of $t$ (i.e., $U=$ $(t-\delta, t+\delta) \cap \mathbb{T}$ for some $\delta>0)$ such that

$$
\left|(f(\sigma(t))-f(s))-f^{\Delta}(t)(\sigma(t)-s)\right| \leq \varepsilon|\sigma(t)-s| \text { for all } s \in U .
$$

The following definitions will be used in the sequel, too.

Definition 2 ([5]). A function $f: \mathbb{T} \rightarrow \mathbb{R}$ is called regulated provided its rightsided limits exist (finite) at right-dense points in $\mathbb{T}$ and its left-sided limits exist (finite) at left-dense points in $\mathbb{T}$. A function $f: \mathbb{T} \rightarrow \mathbb{R}$ is called rd-continuous provided it is continuous at right-dense points in $\mathbb{T}$ and its left-side limits exist (finite) at leftdense points in $\mathbb{T}$.

Definition 3 ([5]). Assume $f: \mathbb{T} \rightarrow \mathbb{R}$ is a regulated function. We define the indefinite integral of a regulated function $f$ by $\int f(t) \Delta t=F(t)+C$, where $C$ is an arbitrary constant and $F$ is a pre-antiderivative of $f$. We define the Cauchy integral by $\int_{a}^{b} f(t) \Delta t=F(b)-F(a)$ for all $a, b \in \mathbb{T}$.

Definition 4 ([5]). We say that a function $p: \mathbb{T} \rightarrow \mathbb{R}$ is regressive provided $1+$ $\mu(t) p(t) \neq 0$ holds for all $t \in \mathbb{T}^{\kappa}$. The set of all regressive and rd-continuous functions $p: \mathbb{T} \rightarrow \mathbb{R}$ is denoted by $\mathcal{R}$. The set of all positively regressive elements of $\mathcal{R}$, is defined as $\mathcal{R}^{+}:=\{p \in \mathcal{R}: 1+\mu(t) p(t)>0$ for all $t \in \mathbb{T}\}$.

An $N \times N$-matrix-valued function $P$ on a time scale $\mathbb{T}$ is called regressive (with respect to $\mathbb{T}$ ) provided

$$
I+\mu(t) P(t) \text { is invertible for all } t \in \mathbb{T}^{\kappa},
$$

where by $I$ we denote the $N \times N$ identity matrix.

Notice that, constant $N \times N$ matrix $P$ is regressive iff the eigenvalues $\lambda_{i}$ of $P$ are regressive for all $1 \leq i \leq N$.

The Grönwall inequality is used in the proof of the main result.

Lemma 1 ([5]). Let $y$ be rd-continuous, $p \in \mathcal{R}^{+}$and $p(t) \geq 0$ for $t \in \mathbb{T}$ and $C \in \mathbb{R}$. Then

implies

$$
y(t) \leq C+\int_{T_{0}}^{t} p(\tau) y(\tau) \Delta \tau \text { for all } t \in \mathbb{T}
$$

$$
y(t) \leq C e_{p}\left(t, T_{0}\right)
$$


Here $e_{p}\left(t, T_{0}\right), T_{0} \in \mathbb{T}$, is a solution of the initial value problem $y^{\Delta}(t)=p(t) y(t)$, $y\left(T_{0}\right)=1$ on $\mathbb{T}$.

Through this paper, assume that

$$
\inf \mathbb{T}=T_{0} \text { and } \sup \mathbb{T}=\infty .
$$

It implies that $\mathbb{T}^{\kappa}=\mathbb{T}$.

\section{SUFFICIENT CONDITIONS FOR CONSENSUS}

\subsection{Adjacency matrix}

The multi-agent system can be modelled by directed or undirected graph. A graph is an object that consists of a non-empty set of vertices and another set of edges. In the graph theory an adjacency matrix is a square matrix used to represent a finite graph. We construct an $N \times N$ adjacency matrix $A$ associated to the graph as follows: if there is an edge from node $i$ to node $j$, then we put 1 as the entry on row $i$, column $j$ of the matrix $A, i, j=1,2, \ldots, N$. Diagonal matrix $D=\operatorname{diag}\left[d_{1}, d_{2}, \ldots, d_{N}\right]$ describes communication between the leader and agents. Entries $d_{i}, i=1,2, \ldots, N$ are positive when there exists information exchange between $i$-th agent and the leader, and $d_{i}=0$ otherwise.

Hermitian matrix (or self-adjoint matrix) is a square matrix that is equal to its own conjugate transpose. Obviously, any symmetric real matrix is Hermitian matrix. If a square matrix equals the multiplication of a matrix and its conjugate transpose, then is Hermitian matrix. The matrix norm induced by the Euclidean norm coincides with the spectral norm and it is submultiplicative norm. Throughout this paper the matrix norm means the spectral norm. If $P$ is an $N \times N$ real matrix, then $\|P\|=$ $\sqrt{\rho\left(P P^{T}\right)}$ where $\rho\left(P P^{T}\right)$ is the largest absolute value of eigenvalues of matrix $P P^{T}$. Moreover, if $P$ is Hermitian, then $\|P\|=\rho(P)$. Symmetric and Hermitian matrices have the property of being always diagonalizable.

Example 1. We consider multi-agent system consisting of the leader and the five agents. In Figure 1 and 2 the topology of the system is given by undirected and directed graph, respectively. The adjacency matrices are the following.

$$
\text { Figure 1: } A=\left[\begin{array}{ccccc}
0 & 1 & 1 & 0 & 0 \\
1 & 0 & 0 & 0 & 0 \\
1 & 0 & 0 & 1 & 1 \\
0 & 0 & 1 & 0 & 0 \\
0 & 0 & 1 & 0 & 0
\end{array}\right] \text {; Figure 2: } A=\left[\begin{array}{ccccc}
0 & 1 & 0 & 0 & 0 \\
1 & 0 & 0 & 0 & 0 \\
1 & 0 & 0 & 0 & 1 \\
0 & 0 & 1 & 0 & 0 \\
0 & 0 & 0 & 0 & 0
\end{array}\right] \text {. }
$$




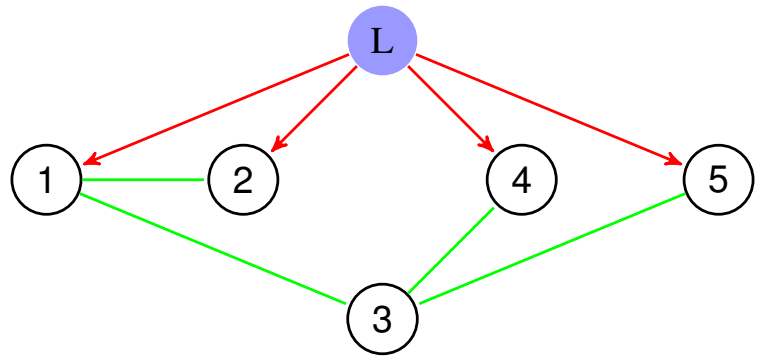

FIGURE 1. The topology of the leader-following multi-agent system under the undirected graph

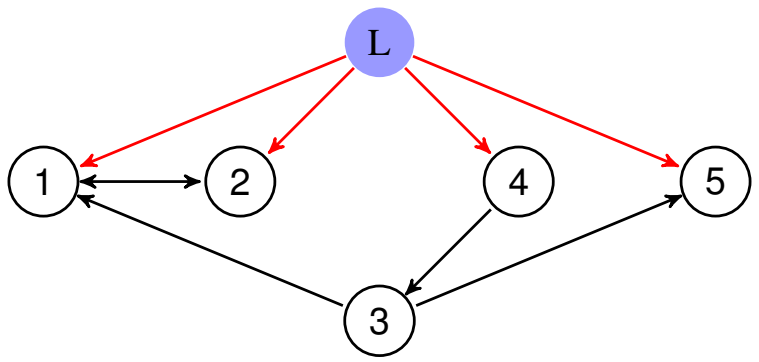

FIGURE 2. The topology of the leader-following multi-agent system under the directed graph

We see that the matrices which represent undirected graph are symmetric. For both graphs we can put for example

$$
D=\left[\begin{array}{lllll}
2 & 0 & 0 & 0 & 0 \\
0 & 2 & 0 & 0 & 0 \\
0 & 0 & 0 & 0 & 0 \\
0 & 0 & 0 & 2 & 0 \\
0 & 0 & 0 & 0 & 2
\end{array}\right]
$$

In this paper, more general adjacency matrix is used which is called weighted adjacency matrix. In weighted adjacency matrix entries are non-negative real numbers. Let $a_{i j}(i, j=1, \ldots, N)$ denote the entries of the weighted adjacency matrix $A$ associated with fixed graph by which the multi-agent systems are modelled. For an undirected graph, $A$ is symmetric. By $L$ we mean the Laplacian matrix $L=\left[l_{i j}\right]$ with $l_{i i}=\sum_{j \neq i} a_{i j}$ and $l_{i j}=-a_{i j}, i, j=1, \ldots, N, i \neq j$. 


\subsection{Mathematical model of agents dynamics}

We consider a discrete time multi-agent system consisting of $N$ agents and the leader. The dynamics of each agent labelled $i, i=1,2, \ldots N$, is given by the following equation

$$
x_{i}^{\Delta}(t)=f\left(t, x_{i}(t)\right)+\gamma \sum_{j=1}^{N} a_{i j}\left(x_{j}(t)-x_{i}(t)\right)+\gamma d_{i}\left(x_{0}(t)-x_{i}(t)\right), t \in \mathbb{T},
$$

where $x_{i}(t)$ represents the state at time $t$ and $\gamma$ is a feedback control gain. Function $f: \mathbb{T} \times \mathbb{R} \rightarrow \mathbb{R}$ describes non-linear dynamics. The leader, labelled as $i=0$, for multi-agent system (3.1) is an isolated agent with trajectory described by

$$
x_{0}^{\Delta}(t)=f\left(t, x_{0}(t)\right), t \in \mathbb{T} .
$$

Notice that the control law $\gamma \sum_{j=1}^{N} a_{i j}\left(x_{j}(t)-x_{i}(t)\right)+\gamma d_{i}\left(x_{0}(t)-x_{i}(t)\right)$ for $i$-th agent used in system (3.1)-(3.2) was studied by many authors including Yu, Jiang and $\mathrm{Hu}$ in [20].

Definition 5. The multi-agent system (3.1)-(3.2) is said to be achieved the leaderfollowing consensus if a solution to (3.1)-(3.2) satisfies

$$
\lim _{t \rightarrow \infty}\left(x_{i}(t)-x_{0}(t)\right)=0, i=1,2, \ldots, N, t \in \mathbb{T},
$$

for any initial conditions $x_{i}\left(T_{0}\right) \in \mathbb{R}, i=0,1,2, \ldots, N, T_{0} \in \mathbb{T}$.

Let us denote by $\varepsilon_{i}(t)=x_{i}(t)-x_{0}(t)$ the distance between the leader and the $i$-th agent. From (3.1)-(3.2) we obtain

$$
\varepsilon_{i}^{\Delta}(t)=f\left(t, x_{i}(t)\right)-f\left(t, x_{0}(t)\right)+\gamma \sum_{j=1}^{N} a_{i j}\left(\varepsilon_{j}(t)-\varepsilon_{i}(t)\right)-\gamma d_{i} \varepsilon_{i}(t)
$$

for $i=1,2, \ldots, N$. Setting

$$
\begin{aligned}
& \varepsilon(t)=\left(\varepsilon_{1}(t), \varepsilon_{2}(t), \ldots, \varepsilon_{N}(t)\right)^{T}, \\
& x(t)=\left(x_{1}(t), x_{2}(t), \ldots, x_{N}(t)\right)^{T}
\end{aligned}
$$

and

$$
\begin{gathered}
F(t, x(t))=\left(f\left(t, x_{1}(t)\right), f\left(t, x_{2}(t)\right), \ldots, f\left(t, x_{N}(t)\right)\right)^{T}, \\
F\left(t, x_{0}(t) \mathbb{1}\right)=\left(f\left(t, x_{0}(t)\right), f\left(t, x_{0}(t)\right), \ldots, f\left(t, x_{0}(t)\right)\right)^{T},
\end{gathered}
$$

system (3.1)-(3.2) takes the following form

$$
\varepsilon^{\Delta}(t)=F(t, x(t))-F\left(t, x_{0}(t) \mathbb{1}\right)-\gamma B \varepsilon(t),
$$

where $B=L+D$ (for proof see [18]). Here $\mathbb{1}$ is the vector $[1, \ldots, 1]^{T}$. 


\subsection{Main results}

We assume that function $f: \mathbb{T} \times \mathbb{R} \rightarrow \mathbb{R}$ satisfies Lipschitz condition with respect to the second variable in the meaning of following definition.

Definition 6. We say that function $f: \mathbb{T} \times \mathbb{R} \rightarrow \mathbb{R}$ fulfills Lipschitz condition if there exists a positive constant $\mathscr{L}$ such that

$$
|f(t, x(t))-f(t, y(t))| \leq \mathscr{L}|x(t)-y(t)|, t \in \mathbb{T} .
$$

Let $P \in \mathcal{R}$. By $e_{P}\left(t, T_{0}\right)$ we denote the unique solution of initial value problem

$$
y^{\Delta}(t)=P y(t), y\left(T_{0}\right)=\mathbb{1} .
$$

So, for regressive $-\gamma B$, function $e_{-\gamma B}\left(t, T_{0}\right)$ is the solution of initial value problem

$$
\varepsilon^{\Delta}(t)=-\gamma B \varepsilon(t), \varepsilon\left(T_{0}\right)=\mathbb{1} .
$$

Theorem 1. Assume that $\mathbb{T}=\mathbb{N}$ and condition (3.4) is satisfied. If

each eigenvalue of matrix $(-\gamma B)$ is regressive with respect to time scale $\mathbb{N}$,

and

$$
\text { spectral norm of matrix }(I-\gamma B) \text { is less than } 1-\mathscr{L} \text {, }
$$

where $\mathscr{L}$ is Lipschitz constant, then system (3.1)-(3.2) achieves the leader-following consensus.

Proof. Condition (3.5) implies that matrix $(-\gamma B) \in \mathcal{R}$.

By $\mathcal{M}$ we denote the spectral norm of matrix $(I-\gamma B)$. Thus, by (3.6), we have

$$
\mathcal{M}+\mathscr{L}<1 .
$$

By variation of constants (see [5]), the unique solution of equation (3.3) with initial condition $\varepsilon\left(T_{0}\right)$, for $t \geq T_{0}$, is given by

$$
\varepsilon(t)=e_{-\gamma B}\left(t, T_{0}\right) \varepsilon\left(T_{0}\right)+\int_{T_{0}}^{t} e_{-\gamma B}(t, \sigma(\tau))\left(F\left(\tau, x_{0}(\tau) \mathbb{1}\right)-F(\tau, x(\tau))\right) \Delta \tau .
$$

Using condition (3.4), we obtain

$$
\|\varepsilon(t)\| \leq\left\|\varepsilon\left(T_{0}\right)\right\|\left\|e_{-\gamma B}\left(t, T_{0}\right)\right\|+\int_{T_{0}}^{t} \mathscr{L}\|\varepsilon(\tau)\|\left\|e_{-\gamma B}(t, \sigma(\tau))\right\| \Delta \tau .
$$

On time scale $\mathbb{T}=\mathbb{N}$ function $e_{-\gamma B}\left(t, T_{0}\right)=(I-\gamma B)^{t-T_{0}}$. This implies

$$
\left\|e_{-\gamma B}\left(t, T_{0}\right)\right\|=\left\|(I-\gamma B)^{t-T_{0}}\right\| \leq\|I-\gamma B\|^{t-T_{0}}=\mathcal{M}^{t-T_{0}} .
$$


From the above and (3.8), putting $\sigma(\tau)=\tau+1$, we get

$$
\|\varepsilon(t)\| \leq\left\|\varepsilon\left(T_{0}\right)\right\| \mathcal{M}^{t-T_{0}}+\int_{T_{0}}^{t} \mathscr{L}\|\varepsilon(\tau)\| \mathcal{M}^{t-\tau-1} \Delta \tau
$$

and

$$
\mathcal{M}^{-t}\|\varepsilon(t)\| \leq\left\|\varepsilon\left(T_{0}\right)\right\| \mathcal{M}^{-T_{0}}+\int_{T_{0}}^{t} \mathscr{L} \mathcal{M}^{-1}\|\varepsilon(\tau)\| \mathcal{M}^{-\tau} \Delta \tau .
$$

By Lemma 1, we obtain

$$
\mathcal{M}^{-t}\|\varepsilon(t)\| \leq\left\|\varepsilon\left(T_{0}\right)\right\| \mathcal{M}^{-T_{0}} e_{\mathscr{L} \mathcal{M}^{-1}}\left(t, T_{0}\right) .
$$

Thus

$$
\|\varepsilon(t)\| \leq\left\|\varepsilon\left(T_{0}\right)\right\| \mathcal{M}^{t-T_{0}}\left(1+\mathscr{L} \mathcal{M}^{-1}\right)^{t-T_{0}}=\left\|\varepsilon\left(T_{0}\right)\right\|(\mathcal{M}+\mathscr{L})^{t-T_{0}} .
$$

Therefore, by (3.7)

$$
0 \leq \lim _{t \rightarrow \infty}\|\varepsilon(t)\| \leq \lim _{t \rightarrow \infty}\left\|\varepsilon\left(T_{0}\right)\right\|(\mathcal{M}+\mathscr{L})^{t-T_{0}}=0 .
$$

It implies the thesis of Theorem 1.

Example 2. Let $\mathbb{T}=\mathbb{N}$. We consider a group of 5 followers and the leader with the following initial conditions: $x_{0}\left(T_{0}\right)=5, x_{1}\left(T_{0}\right)=3, x_{2}\left(T_{0}\right)=14, x_{3}\left(T_{0}\right)=16$, $x_{4}\left(T_{0}\right)=10$ and $x_{5}\left(T_{0}\right)=7$, where $T_{0}=1$, and the symmetric adjacency matrix (see Figure 1) and $D$ given in Example 1. Let $f(x, y)=(0.2 x y) /\left(2+3 x^{2}\right)$ and $\gamma=0.2$. Here $\mathscr{L}=0.2, \mathcal{M}=0,74$. Therefore, by Theorem 1 , the leader-following consensus is achieved. The state trajectories $x_{i}$ and $\varepsilon_{i}, i=1,2,3,4,5$ are shown in Figure 3 and Figure 4, respectively. The trajectory of the leader $x_{0}$ is drown in red and trajectories of $x_{i}, i=1,2,3,4,5$, in green, black, blue, magenta and pink.

Theorem 2. Assume that $\mathbb{T}=h \mathbb{N}, h>0$ and condition (3.4) is satisfied. If each eigenvalue of matrix $(-\gamma B)$ is regressive with respect to time scale $h \mathbb{N}$, and

$$
\text { spectral norm of matrix }(I-h \gamma B) \text { is less than } 1-h \mathscr{L} \text {, }
$$

then the leader-following consensus holds for system (3.1)-(3.2).

Proof. Obviously matrix $(-\gamma B) \in \mathcal{R}$. Here, by $\mathcal{M}^{*}$ we denote spectral norm of matrix $(I-h \gamma B)$. Notice that, if $t, T_{0} \in h \mathbb{N}$ then $\left(t-T_{0}\right) / h \in \mathbb{N}$. We have

$$
e_{-\gamma B}\left(t, T_{a} 0\right)=(I-h \gamma B)^{\frac{t-T_{0}}{h}} \text { and }\left\|e_{-\gamma B}\left(t, T_{0}\right)\right\| \leq\left(\mathcal{M}^{*}\right)^{\frac{t-T_{0}}{h}} .
$$

Applying the same arguments as in the proof of Theorem 1 we get the thesis. 


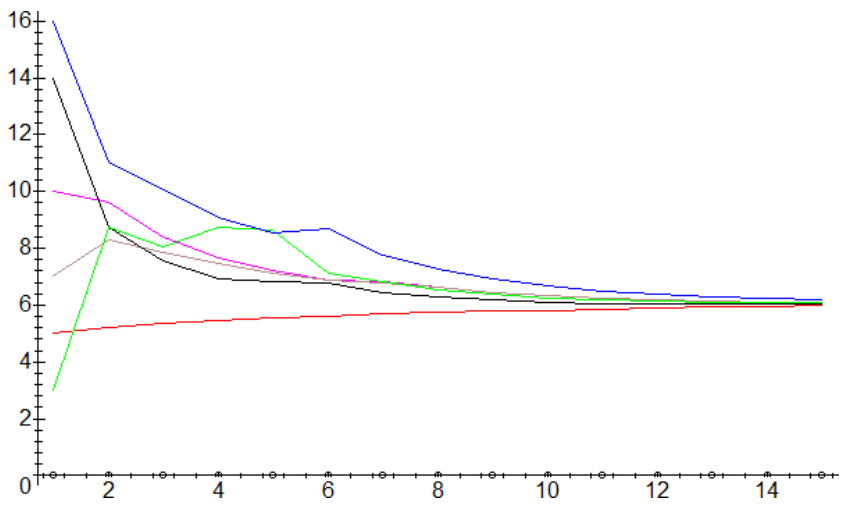

FIGURE 3. Trajectories $x_{i}, i=0,1,2,3,4,5$

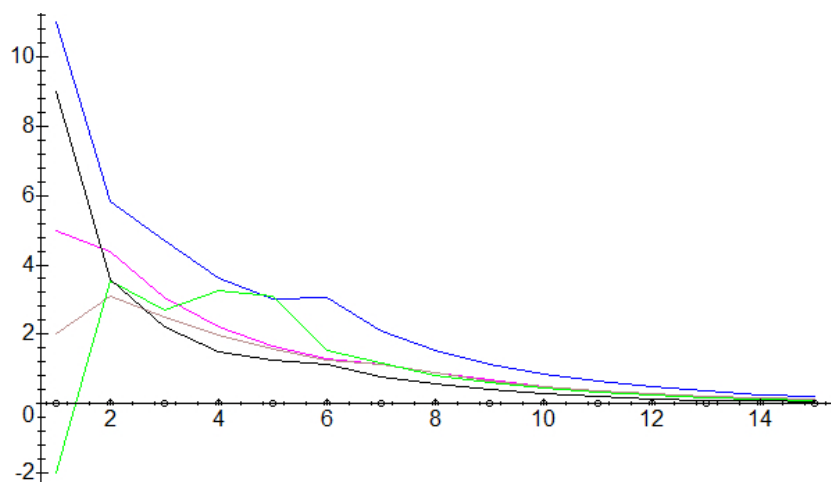

FIGURE 4. Trajectories $\varepsilon_{i}, i=1,2,3,4,5$

Example 3. Now let $\mathbb{T}=0.5 \mathrm{~N}$ and $T_{0}=0.5$. As in Example 2, we consider 5 followers and the leader. We also assume the same initial conditions, the symmetric adjacency matrix, the matrix $D$, the function $f(x, y)=(0.2 x y) /\left(2+3 x^{2}\right)$ and $\gamma=0.2$. Hence, we have $\mathscr{L}=0.2$ and $\mathcal{M} \leq 0.87<1-0.5 \mathscr{L}=0.9$. According to Theorem 2, the group of agents reaches the consensus. The trajectories of the leader and his followers $\left(x_{i}, i=0,1,2,3,4,5\right)$ and distances between them $\left(\varepsilon_{i}, i=1,2,3,4,5\right)$ we draw in Figure 5 and in Figure 6, respectively.

Theorem 3. Assume that time scale $\mathbb{T}$ is discrete and condition (3.4) is satisfied. If

$$
(-\gamma B) \text { is regressive }
$$

and

$$
\text { spectral norm of matrix }(I-\mu(t) \gamma B) \text { is less than } 1-\mu(t) \mathscr{L} \text {, }
$$




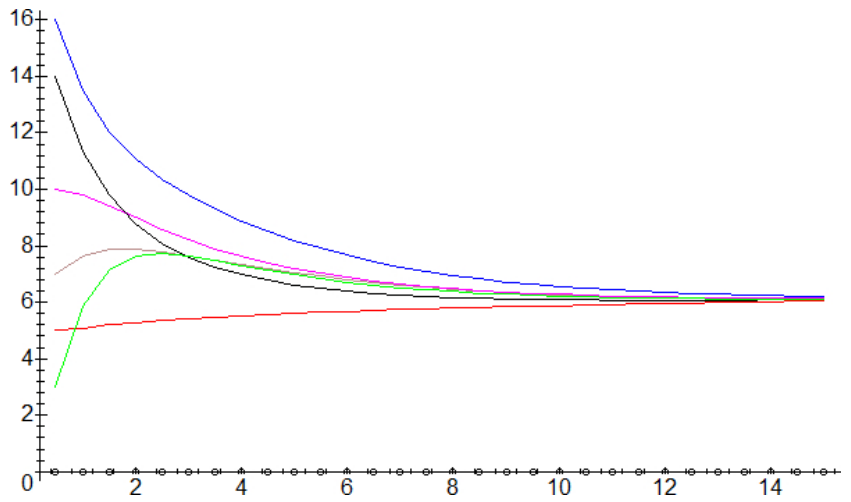

FIGURE 5. Trajectories $x_{i}, i=0,1,2,3,4,5$

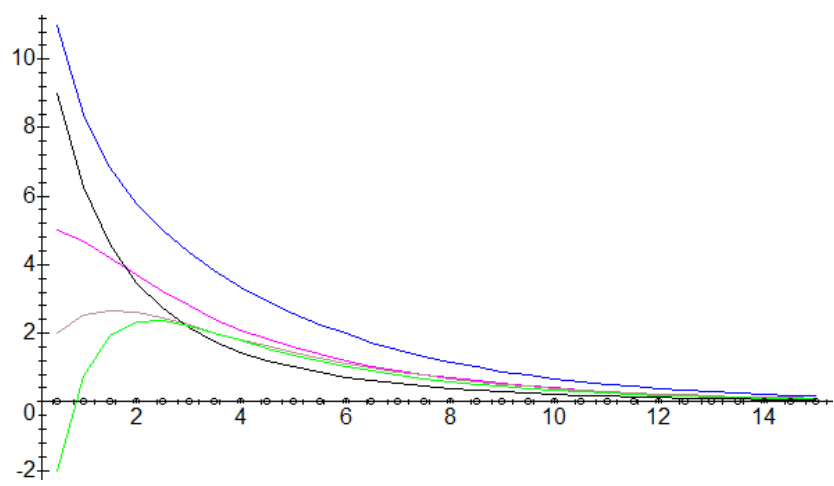

FIGURE 6. Trajectories $\varepsilon_{i}, i=1,2,3,4,5$

then the leader-following consensus holds for system (3.1)-(3.2).

Proof. Let us denote by $\mathcal{M}^{* *}$ supremum of spectral norms of matrices $(I-\mu(t) \gamma B)$. Here

$$
\begin{aligned}
\left\|e_{-\gamma B}\left(t, T_{0}\right)\right\| & =\left\|\prod_{s \in \mathbb{T} \cap\left[T_{0}, t\right)}(I-\mu(s) \gamma B)\right\| \\
& \leq \prod_{s \in \mathbb{T} \cap\left[T_{0}, t\right)}\|(I-\mu(s) \gamma B)\| \leq \prod_{s \in \mathbb{T} \cap\left[T_{0}, t\right)} \mathcal{M}^{* *} .
\end{aligned}
$$


For considered here time scales, inequality (3.8) implies

$$
\|\varepsilon(t)\| \leq\left\|\varepsilon\left(T_{0}\right)\right\| \prod_{s \in \mathbb{T} \cap\left[T_{0}, t\right)} \mathcal{M}^{* *}+\int_{T_{0}}^{t} \mathscr{L}\|\varepsilon(\tau)\| \prod_{s \in \mathbb{T} \cap[\sigma(\tau), t)} \mathcal{M}^{* *} \Delta \tau .
$$

Hence, multiplying the both sides of inequality (3.10) by $\prod_{s \in \mathbb{T} \cap\left(T_{0}, t\right)}\left(\mathcal{M}^{* *}\right)^{-1}$, we obtain

$$
\begin{aligned}
\prod_{s \in \mathbb{T} \cap\left(T_{0}, t\right)}\left(\mathcal{M}^{* *}\right)^{-1}\|\varepsilon(t)\| \leq & \left\|\varepsilon\left(T_{0}\right)\right\| \mathcal{M}^{* *} \\
& +\int_{T_{0}}^{t} \mathscr{L}\left(\mathcal{M}^{* *}\right)^{-1}\|\varepsilon(\tau)\| \prod_{s \in \mathbb{T} \cap\left(T_{0}, \tau\right)}\left(\mathcal{M}^{* *}\right)^{-1} \Delta \tau .
\end{aligned}
$$

By Lemma 1, we obtain

$$
\begin{gathered}
\prod_{s \in \mathbb{T} \cap\left(T_{0}, t\right)}\left(\mathcal{M}^{* *}\right)^{-1}\|\varepsilon(t)\| \leq\left\|\varepsilon\left(T_{0}\right)\right\| \mathcal{M}^{* *} \prod_{s \in \mathbb{T} \cap\left[T_{0}, t\right)}\left(1+\mu(s) \mathscr{L}\left(\mathcal{M}^{* *}\right)^{-1}\right) \\
\|\varepsilon(t)\| \leq\left\|\varepsilon\left(T_{0}\right)\right\| \prod_{s \in \mathbb{T} \cap\left[T_{0}, t\right)}\left(\mathcal{M}^{* *}+\mu(s) \mathscr{L}\right) .
\end{gathered}
$$

By (3.9), we have $\mathcal{M}^{* *}+\mu(s) \mathscr{L}<1$ for any $s \in \mathbb{T}$. Thus

$$
\lim _{t \rightarrow \infty}\|\varepsilon(t)\| \leq \lim _{t \rightarrow \infty}\left\|\varepsilon\left(T_{0}\right)\right\| \prod_{s \in \mathbb{T} \cap\left[T_{0}, t\right)}\left(\mathcal{M}^{* *}+\mu(s) \mathscr{L}\right)=0 .
$$

It implies the thesis.

In the following remark we present the most useful consequence of Theorem 3.

Remark 1. Assume that time scale $\mathbb{T}$ is discrete, graininess function $\mu(t) \in$ $\left\{\mu_{1}, \mu_{2}, \ldots, \mu_{k}\right\}$ and condition (3.4) is satisfied. If for any $i \in\{1,2, \ldots, k\}$ each eigenvalue of matrix $(-\gamma B)$ is regressive with respect to considered time scale, and spectral norm of matrix $\left(I-\mu_{i} \gamma B\right)$ is less than $1-\mu_{i} \mathscr{L}$, then the leader-following consensus holds for system (3.1)-(3.2).

Proof. By $\mathcal{M}^{\max }$ we denote maximum of spectral norms of $\left(I-\mu_{i} \gamma B\right)$ for $i \in$ $\{1,2, \ldots, k\}$. Set $\mu^{*}=\max _{i \in\{1,2, \ldots, k\}} \mu_{i}(t)$. Here

$$
\left\|e_{-\gamma B}\left(t, T_{0}\right)\right\| \leq\left(\mathcal{M}^{\max }\right)^{\left\lfloor\frac{t-T_{0}}{\mu^{*}}\right\rfloor} \text { for } t \geq T_{0} .
$$


Example 4. Consider the time scale

$$
\mathbb{T}=\left\{t: t=\frac{3 n+1}{2} \text { for } n=2 k, t=\frac{3 n}{2} \text { for } n=2 k+1, k \in \mathbb{N} \cup\{0\}\right\},
$$

that is $\mathbb{T}=\{0.5,1.5,3.5,4.5,6.5,7.5, \ldots\}$. In this case we have $T_{0}=0.5$ and $\mu_{1}=1$, $\mu_{2}=2$. Again we observe the behavior of group of 5 followers and the leader with the initial conditions known from the previous examples: $x_{0}\left(T_{0}\right)=5, x_{1}\left(T_{0}\right)=3$, $x_{2}\left(T_{0}\right)=14, x_{3}\left(T_{0}\right)=16, x_{4}\left(T_{0}\right)=10$ and $x_{5}\left(T_{0}\right)=7$. We take the matrix $D$ and the symmetric adjacency matrix given in Example 1. In the numerical computations we use $\gamma=0.1, f(x, y)=(0.1 x y) /\left(2+3 x^{2}\right)$. Hence $\mathscr{L}=0.1$ and

$$
\begin{gathered}
\left\|I-\mu_{1} \gamma B\right\| \leq 0.87<1-\mu_{1} \mathscr{L}=0.9, \\
\left\|I-\mu_{2} \gamma B\right\| \leq 0.74<1-\mu_{2} \mathscr{L}=0.8 .
\end{gathered}
$$

Finally, by Remark 1, the leader-following consensus is also achieved. Below, in Figure 7 we present trajectories of the leader and 5 agents $\left(x_{i}, i=0,1,2,3,4,5\right)$ and in Figure 8 we draw distances between the leader and 5 agents $\left(\varepsilon_{i}, i=1,2,3,4,5\right)$.

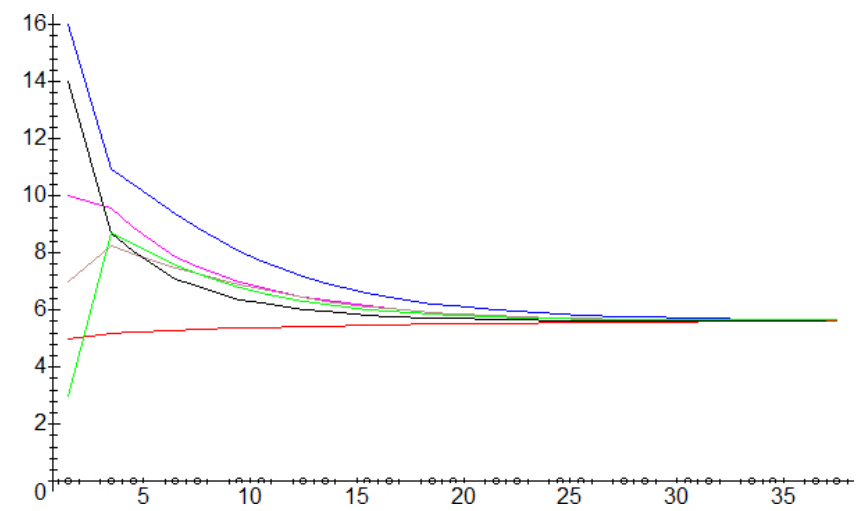

FIGURE 7. Trajectories $x_{i}, i=0,1,2,3,4,5$

Remark 2. If there exists $T^{*} \in \mathbb{T}$ such that time scale considered in Theorem 3 is discrete for $t \geq T^{*}$, then the thesis of this Theorem also holds.

Analogous remarks are valid for Theorems 1 and 2 .

The obtained result generalizes the result obtained by Malinowska, Schmeidel and Zdanowicz in [16] and by Ostaszewska, Schmeidel and Zdanowicz in [17]. In [17], the authors study the leader-following problem on discrete time scales with finite codomain of graininess function. 


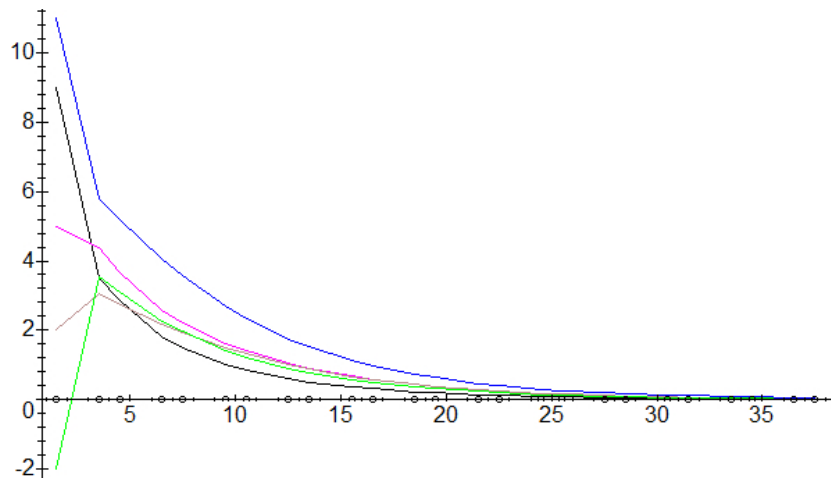

FIGURE 8. Trajectories $\varepsilon_{i}, i=1,2,3,4,5$

\section{CONCLUSION}

In this paper, an emergency of the leader-following consensus of multi-agent systems is investigated on time scales. Based on the stability theory of systems on time scales and Grönwall inequality, sufficient conditions ensuring the leader-following consensus of the model are presented for discrete time scale. Finally, examples are given to demonstrate the effectiveness of the proposed method.

\section{REFERENCES}

[1] B. Aulbach and S. Hilger, "A unified approach to continuous and discrete dynamics," in Qualitative theory of differential equations, A. North-Holland, Ed., doi: 10.1109/IYCE.2015.7180798, 1990.

[2] S. Babenko, M. Defoort, M. Djemai, and S. Nicaise, "On the consensus tracking investigation for multi-agent systems on time scale via matrix-valued Lyapunov functions," Automatica, vol. 87, pp. 316-326, 2018, doi: 10.1016/j.automatica.2018.08.003.

[3] V. D. Blondel, J. M. Hendrickx, and J. N. Tsitsikli, "On Krause's multi-agent consensus model with state-dependent connectivity," IEEE Transactions on automatics control, vol. 54, no. 11, pp. 2586-2597, 2009, doi: 10.1109/TAC.2009.2031211.

[4] V. D. Blondel, J. M. Hendrickx, and J. N. Tsitsikli, "Continuous-time average-preserving opinion dynamics with opinion-dependent communications," SIAM J. Control Optim., vol. 18, no. 8, pp. 5214-5240, 2010, doi: 10.1137/090766188.

[5] M. Bohner and A. Peterson, Dynamic equations on time scales. Birkhäuser, 2001.

[6] M. Bohner and A. Peterson, Advances in dynamic equations on time scales. Birkhäuser, 2003.

[7] F. Cucker and S. Smale, "Emergent behavior in flocks," IEEE Transactions on Automatic Control, vol. 52, no. 7, pp. 852-862, 2007, doi: 10.1109/TAC.2007.895842.

[8] F. Cucker and S. Smale, "On the mathematics of emergence," Japanese Journal of Mathematics, vol. 2, no. 1, pp. 197-227, 2007, doi: 10.1007/s11537-007-0647-x.

[9] M. H. DeGroot, "Reaching a consensus," Journal of the American Statistical Association, vol. 69, pp. 118-121, 1974, doi: 10.1080/01621459.

[10] J. French, "A formal theory of social power," Social Networks, A Developing Paradigm, pp. 35-48, 1977, doi: 10.1016/B978-0-12-442450-0.50010-9. 
[11] E. Girejko, L. Machado, A. B. Malinowska, and N. Martins, "Krause's model of opinion dynamics on isolated time scales," Mathematical Methods in the Applied Sciences, vol. 39, no. 18, pp. 53025314, 2016, doi: 10.1002/mma.3916.

[12] E. Girejko, L. Machado, A. B. Malinowska, and N. Martins, "On consensus in the Cucker-Smale type model on isolated time scale," Discrete and Continuous Dynamical Systems, vol. 11, no. 1, pp. 77-89, 2018, doi: 10.3934/dcdss.2018005.

[13] E. Girejko, A. Malinowska, E. Schmeidel, and M. Zdanowicz, "The emergence on isolated time scales," in 21st International Conference on Methods and Models in Automation and Robotics (MMAR), IEEExplore, Ed., doi: 10.1109/MMAR.2016.7575317, 2016.

[14] A. Jadbabaie, J. Lin, and A. S. Morse, "Coordination of groups of mobile autonomous agents using nearest neighbor rules," IEEE Transactions on Automatic Control, vol. 48, no. 6, pp. 988-1001, 2003, doi: 10.1109/TAC.2003.812781.

[15] U. Krause, "A discrete nonlinear and non-autonomous model of consensus formation," in Comunications in Difference Equations, Gordon and B. Publ., Eds., doi: 10.1109/IYCE.2015.7180798, 2000.

[16] A. B. Malinowska, E. Schmeidel, and M. Zdanowicz, "Discrete leader-following consensus," Mathematical Methods in the Applied Sciences, vol. 40, no. 18, pp. 7307-7315, 2017, doi: 10.1002/mma.4530.

[17] U. Ostaszewska, E. Schmeidel, and M. Zdanowicz, "Leader-following consensus on discrete time scales," in AIP Confonference Proceedings, A. I. of Physics, Ed., doi: 10.1063/1.5044162, 2018.

[18] E. Schmeidel, "The existence of consensus of a leader-following problem with Caputo fractional derivative," Opuscula Mathematica, vol. 39, no. 1, pp. 77-89, 2019, doi: 10.1016/B978-0-12442450-0.50010.

[19] Y. Wang, L. Cheng, H. Wang, Z. G. Hou, M. Tan, and H. Yu, "Leader-following consensus of discrete-time linear multi-agent systems with communication noises," in Lecture Notes in Electrical Engineering 407, IEEE, Ed., doi: 10.1109/ChiCC.2015.7260748, 2015.

[20] Z. Yu, H. Jiang, and C. Hu, "Leader-following consensus of fractional-order multiagent systems under fixed topology," Neurocomputing, vol. 149, pp. 613-620, 2015, doi: 10.1016/j.neucom.2014.08.013.

Authors' addresses

Ewa Schmeidel

University of Bialystok, Institute of Mathematics, 1M Ciołkowskiego St., 15245 Białystok, Poland

E-mail address: eschmeidel@math.uwb.edu.pl

Urszula Ostaszewska

University of Bialystok, Institute of Mathematics, 1M Ciołkowskiego St., 15245 Białystok, Poland

E-mail address: uostasze@math.uwb.edu.pl

Małgorzata Zdanowicz

University of Bialystok, Institute of Mathematics, 1M Ciołkowskiego St., 15245 Białystok, Poland

E-mail address: mzdan@math.uwb.edu.pl 